1 Universidade Federal da Bahia (UFBA) - Salvador (BA), Brasil.

therezacoelho@gmail.com

\section{Representações sociais de doença, usos e significados atribuídos às Práticas Integrativas e Complementares por universitários}

\author{
Social representations of disease, uses and meanings attributed to \\ Integrative and Complementary Practices by students
}

Maria Thereza Ávila Dantas Coelho', Vinicius Pereira de Carvalho', Carle Porcino'

DOI: 10.1590/0103-1104201912215

RESUMO O objetivo deste estudo foi apreender as representações sociais de estudantes universitários sobre a doença e analisar os usos e significados conferidos por eles às Práticas Integrativas e Complementares. Para isso, realizou-se uma pesquisa qualitativa apoiada na Teoria das Representações Sociais, utilizando um questionário semiestruturado para a coleta dos dados, que incluiu, em sua estrutura, o Teste de Associação Livre de Palavras. A maior parte dos estudantes declarou estar no primeiro semestre do curso, na faixa etária de 18 a 24 anos e possuir religião. Para eles, a doença esteve mais associada ao desequilíbrio, sendo a massagem a prática mais adotada. Esses estudantes relacionaram as práticas integrativas à produção de benefícios à vida, à saúde e à manutenção ou alcance da qualidade de vida individual. Nesse sentido, as respostas sugerem que os discentes acreditam na eficácia das práticas integrativas, considerando-as como ligadas à promoção, prevenção e recuperação da saúde. Eles mostram-se abertos a outras racionalidades médicas, ainda que também estejam ligados ao modelo biomédico. Compreender como estudantes pensam a doença e a utilização de práticas integrativas colabora para atribuir sentidos aos projetos terapêuticos organizados no sistema de saúde, dirigindo-se a atenção para a construção da integralidade do cuidado.

PALAVRAS-CHAVE Doença. Terapias complementares. Estudantes. Universidades.

ABSTRACT The objective of this study was to apprehend the social representations of university students about disease and to analyze the uses and meanings conferred by them to Integrative and Complementary Practices. For this purpose, a qualitative research based on the Theory of Social Representations was carried out, using a semi-structured questionnaire to collect the data, which included, in its structure, the Free Word Association Test. The majority of students declared to be attending the first semester of the course, in the age group of 18 to 24 years old and has a religion. For them, disease was most associated with imbalance, with massage being the practice most adopted. These students have related integrative practices to production of benefits to life, health and maintenance or achievement of individual quality of life. In this sense, the answers suggest that students believe in the efficacy of integrative practices, considering them as linked to health's 
promotion, prevention and recovery. They are open to other medical rationalities, although they are also linked to the biomedical model. Understanding how students think about disease and use of integrative practices collaborate to assign meanings to therapeutic projects organized in health system, directing the health care to construction of comprehensive care.

KEYWORDS Disease. Complementary therapies. Students. Universities.

\section{Introdução}

Inúmeras são as formas de representar a doença em uma mesma sociedade, pois existem variações de pensamentos entre grupos e indivíduos. Todavia, uma ideia sempre se afirma hegemônica em relação às outras num dado contexto cultural ${ }^{1-3}$. No mundo ocidental, as representações dominantes de doença estão associadas às racionalidades médicas dominantes, mesmo que sofram a influência das concepções populares ${ }^{2}$. Para Canguilhem ${ }^{4}$, entretanto, é o indivíduo que tem o poder de dizer em qual grau de normalidade se encontra, pois somente ele é capaz de distinguir onde se inicia uma patologia. Por conseguinte, a normalidade e os fenômenos patológicos em si apresentam caráter individual, e o que pode ser considerado para um organismo como anormal, para outro, pode ser normal.

De modo diverso, para Durkheim ${ }^{5}$, as representações estão presentes nos pensamentos individuais e são impostas pelo pensamento coletivo. Nessa direção, Moscovici ${ }^{6}$ considerou que as representações são sociais e manifestam-se por meio das expressões do sujeito individual ou coletivo nas interações sociais, constituindo-se como parte integrante da sociedade e, mais amplamente, do contexto social em que emergem. Inseridas nos ideais contemporâneos, continuamente em transformação, elas são dinâmicas, compartilhadas por grupos, referem-se a um objeto comum e possuem 3 dimensões: informação (conhecimentos sobre o objeto representado), campo da representação (opiniões) e atitude (posicionamento do sujeito) ${ }^{6,7}$. Assim, elas implicam a influência mútua entre o sistema social e o sujeito ${ }^{6,8}$, havendo a necessidade da ação humana para a sua manifestação ${ }^{9}$.

Desse modo, as representações sociais são criadas sempre que um novo saber é elaborado e compartilhado, alcançando o campo de concepções e práticas dos grupos, de forma que os sujeitos pertencentes a eles irão construir e adquirir as representações ao entrarem em contato com as ideias e/ou os novos achados ${ }^{6,7}$. Por esse ângulo, as representações sociais estimulam a preocupação com relação às atitudes ideológicas dos grupos sociais, pois são estas que subscrevem a organização das estruturas que compõem a sociedade ${ }^{6}$, presentes também no campo da saúde. Assim, os estudos sobre representações sociais de saúde e doença são relevantes para a saúde coletiva ${ }^{2,9}$.

Nesse sentido, o corpo social possui representações acerca da cura das doenças, que envolvem os discursos, os sistemas médicos dominantes e os dominados, nos quais estão inseridas as Práticas Integrativas e Complementares (PIC), ainda com pouca força no mundo ocidental' 1 . Entretanto, a desassistência em saúde, pelos sistemas médicos dominantes, tem contribuído para o crescimento das $\mathrm{PIC}^{10}$, uma vez que estas contrapõem-se à natureza generalizante, mecanicista e analítica da biomedicina, que detém a hegemonia no campo da saúde ocidental ${ }^{10,11}$. A racionalidade biomédica caracteriza-se, portanto, por seguir um modelo cartesiano, que situa a medicina 
enquanto ciência das doenças, destituindo a práxis de sua dimensão humanizada e sua apresentação enquanto arte (de curar) ${ }^{\mathbf{1 0}, 11}$.

Como disposto na Política Nacional de Práticas Integrativas e Complementares (PNPIC), as PIC envolvem sistemas médicos complexos e recursos terapêuticos ${ }^{\mathbf{1 2}}$ que apresentam uma abordagem holística do processo saúde-doença-cuidado, intervindo tanto na promoção e recuperação da saúde quanto na prevenção de doenças e agravos. Além disso, proporcionam a possibilidade de maior exercício de cidadania em saúde, resultam em menores custos para o sistema de saúde e integram o ser humano ao meio sociocultural, promovendo o autocuidado e a escuta acolhedo$\mathrm{ra}^{12}$. À vista disso, as PIC apresentam-se como potencializadoras do arranjo de trabalho em equipes multiprofissionais de saúde com centralidade do cuidado no usuário ${ }^{13}$. Esses aspectos se coadunam com os princípios do Sistema Único de Saúde (SUS), tais como a integralidade da assistência ${ }^{14}$.

No Brasil, essas práticas foram integradas ao eixo de cuidado do SUS oficialmente em 2006, por intermédio da PNPIC. Na sua publicação, abrangia medicina tradicional chinesa, homeopatia, medicina antroposófica, termalismo e fitoterapia ${ }^{\mathbf{1 2}}$. No entanto, em 2017 e 2018, houve a ampliação dessa oferta, com a inclusão de 24 novas práticas, 14 delas em 2017 e 10 em 2018 15,16.

Embora já dispostas no âmbito do SUS, as PIC apresentam diversas dificuldades de implementação e difusão. Isso é materializado pelo fato de os cursos superiores da área de saúde ainda apresentarem barreiras para incluí-las em seus currículos formais, provocando a existência de espaço reduzido para a sua discussão ${ }^{17-22}$. Nesse sentido, estudantes de medicina, por exemplo, têm apresentado conhecimentos científicos insuficientes sobre as PIC17,21,23, além de revelarem desinteresse em utilizá-las em seu trabalho futuro $^{21}$. Tais constatações podem se constituir em um grande problema, tendo em vista que é no ambiente acadêmico que os futuros profissionais de saúde aprendem e apreendem os conhecimentos que adotarão nas práticas de cuidado. Sendo assim, se apenas a biomedicina é aprendida, provavelmente, somente ela será utilizada no momento do (des)encontro terapêutico.

Enquanto isso, acadêmicos de saúde têm reconhecido nas PIC um caminho alternativo e eficaz para a promoção da saúde, o tratamento e a prevenção de doenças. Prova disso é a grande aprovação por parte de discentes da inserção da abordagem sobre as PIC nos seus respectivos cursos ${ }^{19,21,24-27}$, corroborando os estudos que apontam a necessidade imediata da oferta de componentes curriculares que comportem essa temática ${ }^{17,19,20,22}$. Além disso, a PNPIC ressalta a necessidade de inserção dessas práticas nos cursos universitários, e a educação superior em saúde deve tornar as pessoas aptas a manejá-las.

Por conseguinte, a relevância deste estudo decorre da necessidade de se produzir conhecimento sobre tais aspectos emergentes, fornecendo subsídios para o fortalecimento de políticas públicas. A doença e as PIC foram compreendidas aqui, portanto, como associadas às experiências individuais e coletivas, inseridas em contextos socioculturais, mas não dissociadas de processos biológicos ${ }^{28}$. Ademais, a discussão sobre o que pensam os estudantes da área de saúde sobre as doenças e as PIC integra a atuação dos profissionais de saúde - também cuidadores de si e de seus iguais -, favorecendo um cuidado equânime, eficaz e atuante, que considere singularidades e especificidades. Nesse sentido, este trabalho objetivou apreender as representações sociais acerca da doença entre estudantes do Bacharelado Interdisciplinar em Saúde (BIS) da Universidade Federal da Bahia (UFBA) e compreender os usos e significados atribuídos às PIC por eles.

\section{Métodos}

Trata-se de uma pesquisa de abordagem qualitativa, fundamentada na Teoria das Representações Sociais (TRS), desenvolvida por Serge Moscovici ${ }^{6}$. Essa abordagem mostra-se pertinente nos estudos relacionados à saúde, uma vez que as relações sociais, os processos 
de cuidado, a estrutura dos sistemas e serviços de saúde e os princípios formativos de recursos humanos em saúde são carregados de representações que conferem autoridade aos discursos que permeiam o campo da saúde. Nesse sentido, as representações sociais constituem-se como pensamentos que guiam a sociedade em sua ação sobre si e sobre o mundo ${ }^{6}$.

Participaram voluntariamente deste estudo 223 estudantes do BIS da UFBA. Foram definidos como critérios de inclusão: ser aluno do BIS e possuir no mínimo 18 anos de idade. Nesse sentido, 243 pessoas participaram da coleta de dados, mas 20 foram excluídas por fazerem outro curso. Para a coleta de dados, foi utilizado um questionário semiestruturado, com questões ligadas aos dados sociodemográficos, às representações de doença, à utilização e aos significados atribuídos às PIC. Esse questionário incluiu, em sua estrutura, o Teste de Associação Livre de Palavras (Talp), para apreender as representações sobre a doença. Para captar os usos e significados conferidos pelos participantes às PIC, foram analisadas as respostas das seguintes perguntas do questionário: 'o que vocêfaz quando está doente?', 'qual(is) são as práticas abaixo que você utiliza ou já utilizou?' e 'independentemente de adotar ou não essas práticas, que significados elas têm para você?'. Esse instrumento foi aplicado no período de 15 a 31 de maio de 2017, durante as aulas do componente curricular obrigatório 'Introdução ao campo da saúde'.

A técnica da Associação Livre de Palavras, desenvolvida por Jung ${ }^{29}$, foi adaptada por Di Giacomo ${ }^{30}$ para o campo da psicologia social. Desde então, tem sido largamente empregada em pesquisas sobre representações sociais ${ }^{31}$. O Talp, enquanto uma técnica projetiva, é útil para revelar as representações acerca de objetos e fenômenos, pois acessa a organização psíquica dos sujeitos, trazendo à tona para o pesquisador o que está oculto ou não $0^{32}$. No presente estudo, o termo indutor foi 'doença', para o qual foi solicitado que se evocasse até cinco palavras ou expressões curtas que viessem imediatamente à consciência, em associação ao termo. Por fim, entre as palavras escritas, solicitou-se que assinalassem aquela considerada como sendo a mais importante.

No processamento dos termos evocados acerca do estímulo doença, foi utilizada a análise textual com o auxílio do software Iramuteq, que conformou uma nuvem de palavras, em que é possível observar, por meio do tamanho e da espessura dos termos, a frequência simples, de modo que quanto maior é o tamanho da fonte, maior é a representatividade do termo no corpus em análise ${ }^{33}$.

No que se refere aos usos das PIC, utilizou-se o software IBM SPSS Statistics, que pôde fornecer um gráfico com a utilização dessas práticas ${ }^{\mathbf{3 4}}$. $\mathrm{O}$ corpus constituído a partir dos significados atribuídos às PIC foi organizado e processado com o auxílio do software Iramuteq, que gerou a Classificação Hierárquica Descendente (CHD). Na constituição da CHD, o corpus foi organizado em Unidades de Contexto Elementar (UCE), que são os menores fragmentos de texto com sentido das respostas, e separado em classes, nas quais os elementos que as compõem apresentam certa homogeneidade entre $\mathrm{si}^{35}$. Para a apresentação da CHD, foi constituído um dendograma formado pelos termos mais significativos que surgiram nas respostas agrupadas em cada classe.

Seguindo a orientação de Herzlich ${ }^{8}$, a análise dos dados buscou situar as representações dos sujeitos nos planos ideológico, social e histórico em que estão inseridas. Delineou-se, então, como se caracterizam as representações e em quais modelos sociais estão inseridas ${ }^{9}$, associando-as às práticas que as determinam ou são determinadas por elas ${ }^{\mathbf{2 8}}$.

Os participantes assinaram o Termo de Consentimento Livre e Esclarecido (TCLE), e o projeto foi aprovado pelo Comitê de Ética em Pesquisa da Escola de Enfermagem da UFBA, sob o Parecer $n^{0} 741.187$, respeitando a Resolução $n^{0} 466 / 2012^{36}$. Com o propósito de assegurar o anonimato dos participantes, as respostas estão identificadas no texto alfanumericamente, configuradas no seguinte termo: (número do questionário-gênero-idade). 


\section{Resultados e discussão}

\section{Perfil sociodemográfico dos participantes}

Dos 223 participantes deste estudo, 138 se autorreferiram como mulheres, 83 como homens, um como 'outros' e uma pessoa optou por não declarar a autorreferência. A maioria $(51,1 \%)$ se encontrava no primeiro semestre, na faixa etária entre 18 e 24 anos, sendo pardos ou pretos. É importante destacar que, para $70,40 \%$ dos estudantes ingressantes, o BIS é a primeira graduação. Isso corrobora a proposta política e pedagógica dos Bacharelados Interdisciplinares, os quais pretendem constituir o primeiro de um regime de três ciclos, sendo, portanto, a porta de entrada para os estudantes na Universidade ${ }^{37}$. Ademais, $57,84 \%$ afirmaram ser baianos e adeptos de alguma religião (tabela 1).

Tabela 1. Caracterização sociodemográfica dos estudantes do Bacharelado Interdisciplinar em Saúde da UFBA, que informaram o gênero. Salvador, Bahia, Brasil, 2017 ( $n=221)$

\begin{tabular}{|c|c|c|c|c|c|c|}
\hline \multirow[t]{2}{*}{ Variável } & \multicolumn{2}{|r|}{ Homem } & \multicolumn{2}{|r|}{ Mulher } & \multicolumn{2}{|r|}{ Total } \\
\hline & $\mathbf{N}$ & $\%$ & $\mathbf{N}$ & $\%$ & $\mathrm{~N}$ & $\%$ \\
\hline \multicolumn{7}{|l|}{ Faixa etária } \\
\hline 18 a 24 anos & 64 & $28,69 \%$ & 111 & $49,77 \%$ & 175 & $78,47 \%$ \\
\hline 25 a 32 anos & 13 & $5,82 \%$ & 17 & $7,62 \%$ & 30 & $13,44 \%$ \\
\hline 32 a 39 anos & 5 & $2,24 \%$ & 4 & $1,79 \%$ & 9 & $4,03 \%$ \\
\hline Acima de 40 anos & 0 & $0,00 \%$ & 3 & $1,34 \%$ & 3 & $1,34 \%$ \\
\hline Não respondeu & 1 & $0,44 \%$ & 3 & $1,34 \%$ & 4 & $1,78 \%$ \\
\hline \multicolumn{7}{|l|}{ Cor autodeclarada } \\
\hline Branca & 15 & $6,72 \%$ & 33 & $14,79 \%$ & 48 & $21,51 \%$ \\
\hline Preta & 24 & $10,76 \%$ & 24 & $10,76 \%$ & 48 & $21,52 \%$ \\
\hline Parda & 40 & $17,93 \%$ & 78 & $34,97 \%$ & 118 & $52,91 \%$ \\
\hline Amarela & 4 & $1,79 \%$ & 3 & $1,34 \%$ & 7 & $3,13 \%$ \\
\hline \multicolumn{7}{|l|}{ Tem religião? } \\
\hline Sim & 45 & $20,17 \%$ & 100 & $44,84 \%$ & 145 & $65,01 \%$ \\
\hline Não & 38 & $17,04 \%$ & 38 & $17,04 \%$ & 76 & $34,08 \%$ \\
\hline \multicolumn{7}{|l|}{ Naturalidade } \\
\hline Salvador & 40 & $17,93 \%$ & 68 & $30,49 \%$ & 108 & $48,43 \%$ \\
\hline Interior da Bahia & 38 & $17,04 \%$ & 53 & $23,76 \%$ & 91 & $40,80 \%$ \\
\hline Fora da Bahia & 4 & $1,79 \%$ & 15 & $6,72 \%$ & 19 & $8,51 \%$ \\
\hline Não respondeu & 1 & $0,44 \%$ & 2 & $0,89 \%$ & 3 & $1,33 \%$ \\
\hline \multicolumn{7}{|l|}{ Semestre } \\
\hline Ingresso & 70 & $31,39 \%$ & 115 & $51,56 \%$ & 185 & $82,95 \%$ \\
\hline Não ingresso & 13 & $5,82 \%$ & 23 & $10,31 \%$ & 36 & $16,13 \%$ \\
\hline
\end{tabular}

Fonte: Elaboração própria. 
A participante que se autorreferiu como 'outros' e a que optou por não responder a 'autorreferência' de gênero pertenciam à faixa etária de 18 a 24 anos, declararam cor parda, não ter religião, ser natural de Salvador e estar no primeiro semestre.

Quanto à questão religiosa, 65,01\% dos estudantes referiram ser adeptos de alguma religião, 49,65\% eram católicos, 28,96\% protestantes, $14,48 \%$ espíritas, $3,44 \%$ de religiões de matriz africana e 3,44\% relataram frequentar/participar de outras doutrinas ou seitas.

\section{Representações da doença}

Na aplicação do Talp, foram evocadas 1050 palavras, das quais, 77 eram diferentes (figura 1).

Figura 1. Nuvem de palavras processada através do software Iramuteq, referente às evocações realizadas pelos estudantes do BIS da UFBA acerca do termo indutor 'doença'. Salvador, Bahia, Brasil, 2017

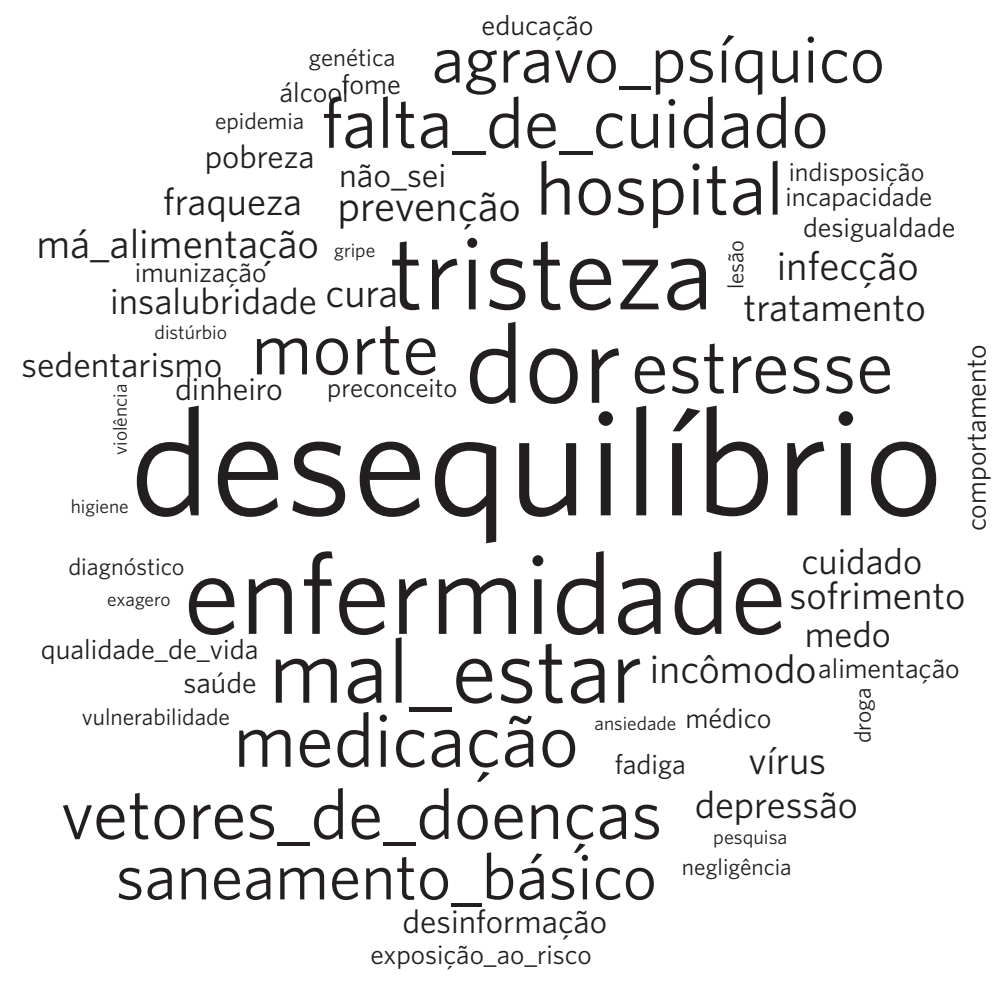

Fonte: Elaboração própria.

Entre os termos mais evocados, desequilíbrio ocupa a primeira posição ( $\mathrm{F}=83)$, seguido de dor (63), enfermidade (61), tristeza (57), mal-estar (47), hospital (40), morte (40), estresse (40), medicação (37), falta de cuidado (35), vetores de doença (33), agravo psíquico (32), saneamento básico (30), fadiga (19) e prevenção (18). Essa representação está associada ao conhecimento corrente, socialmente construído ${ }^{6}$, ou seja, os estudantes expõem suas opiniões ordenadas a partir das interações sociais estabelecidas dentro e fora da Universidade. 
A concepção de doença como 'desequilíbrio’ também aparece como dominante em um estudo realizado com estudantes da primeira turma do BIS da UFBA durante o $1^{\circ}$ ano de estudos, em 200938. Além de conceberem a doença como desequilíbrio, observa-se que ela é representada como negativa, pois provoca 'dor', 'tristeza', 'mal-estar' e 'morte'. Entre os termos evocados, fica evidente que a experiência patológica foi considerada nociva, em consonância ao saber médico ocidental, que simboliza a doença como uma anormalidade contraproducente ${ }^{\mathbf{1}}$. Esses achados corroboram a compreensão de que esse grupo formado por estudantes majoritariamente ingressantes na universidade apresenta uma representação social da doença relacionada aos sistemas médicos dominantes.

Para os participantes, o tratamento dispensado às condições de adoecimento dar-se-á por meio do que está disposto pelo modelo biomédico, como, por exemplo, dirigir-se ao hospital e/ou (auto)medicar-se. A racionalidade homeopática não faz parte desse leque de cuidados, e a doença, assim como seus sintomas, deve ser combatida e ter um fim em si mesma ${ }^{1}$. Seguindo essa ideia, é apontado que a doença pode ser prevenida, na medida em que os termos 'vetores de doença', 'saneamento básico’ e 'prevenção’ são evocados.

Além disso, tais representações estão associadas às doenças mentais, haja vista a presença de evocações referentes a 'agravo psíquico'. Isso pode ser atribuído à prevalência de doenças no âmbito da saúde mental na contemporaneidade, relacionada ao fenômeno da medicalização da vida, que abarca em seu escopo a patologização de comportamentos, presente no campo da educação $0^{39}$. Tomando como base as ideias de Canguilhem ${ }^{4}$, entretanto, é possível visualizar que as alterações de padrões de normalidade podem não se estabelecer como doenças ou ser, em si mesmas, vias para a manutenção ou o estabelecimento de uma nova normalidade.

\section{Usos de Práticas Integrativas e Complementares}

No que se refere à experiência pessoal com as PIC, $41,57 \%$ dos estudantes responderam que as utilizavam quando estavam doentes, sendo apontadas como as mais usadas por $16,14 \%$. Ao mesmo tempo, $85,65 \%$ procuram um profissional de saúde, $68,16 \%$ automedicação, 64,57\% remédios prescritos, 17,69\% tratamentos religiosos e $15,69 \%$ outras práticas. Essa porcentagem de uso das PIC entre estudantes de saúde está conforme a literatura sobre o tema. No estudo de Morales et al. ${ }^{18} \mathrm{com}$ estudantes de medicina, por exemplo, somente $30,6 \%$ relataram ter utilizado PIC em algum momento da vida, ao passo que no trabalho de Contreras et al. ${ }^{25}$ essa porcentagem foi de $49 \%$. É possível depreender, portanto, que a atitude referente ao uso das PIC pelos participantes desta pesquisa ratifica as suas representações sociais sobre doença: ainda que tal utilização seja expressiva, ela é minoritária em relação à utilização da medicina tecnocientífica. $\mathrm{O}$ modelo biomédico domina, assim, tanto as representações sobre doença quanto as práticas de cuidado desse grupo.

Independentemente do motivo de uso, a massagem aparece como a PIC mais utilizada (115 estudantes indicaram seu uso) (gráfico 1). 
Gráfico 1. Usos das Práticas Integrativas e Complementares pelos estudantes do BIS da UFBA, processado através do software IBM SPSS Statistics. Salvador, Bahia, Brasil, 2017 ( $n=223)$

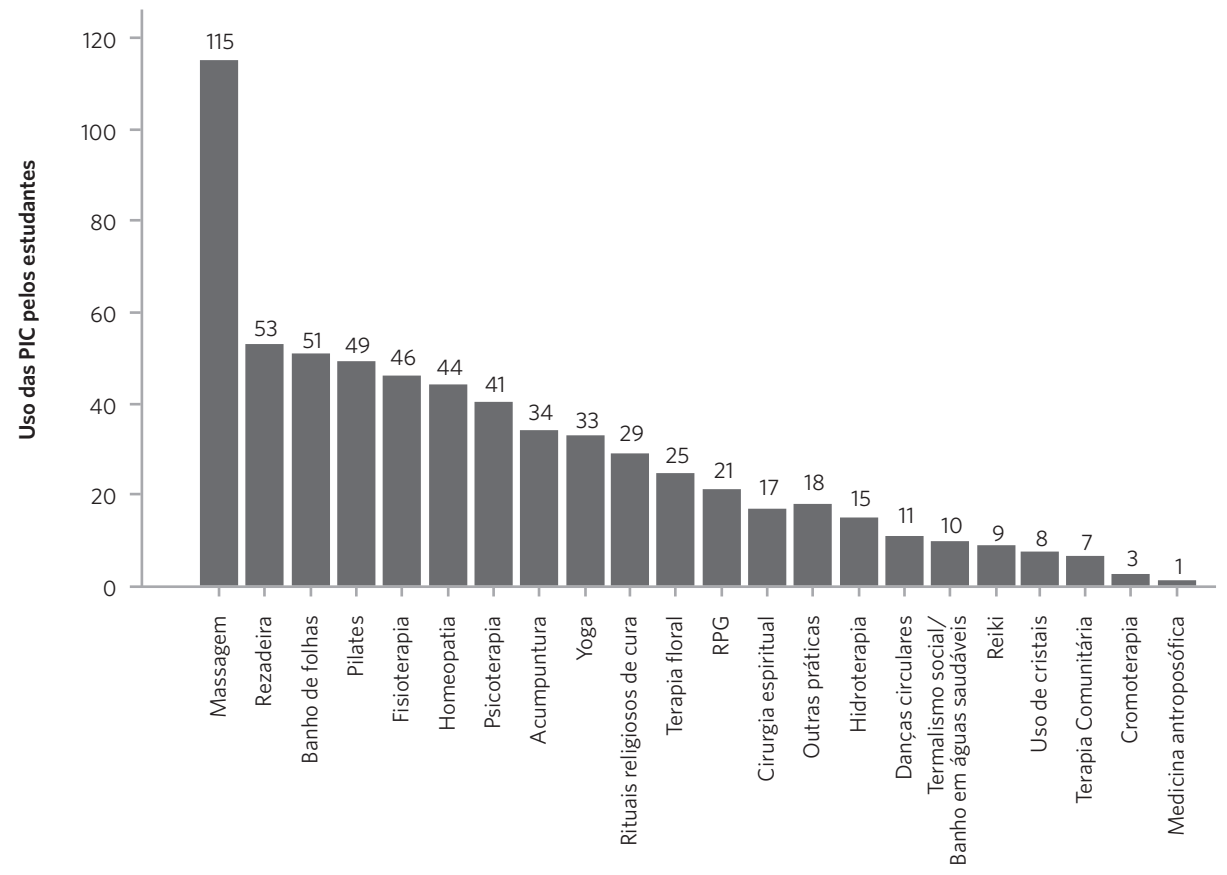

Fonte: Elaboração própria.

A utilização da massagem, referida pelos participantes, pode refletir a difusão de terapêuticas alternativas na sociedade, que visam a dar conta de problemas considerados menos ‘importantes' ou com baixos 'danos à saúde', persistindo os de maior complexidade a serem tratados nos campos da medicina hegemônica. Em estudo de $\mathrm{Luz}^{3}$, os demandantes do cuidado acreditavam que uma dor somente se configura como doença quando os incapacita de realizar as tarefas diárias, incluindo aquelas relacionadas ao trabalho. Assim, se uma dor não leva a uma incapacidade funcional, não precisa ser tratada formalmente pelo saber médico instituído, sendo utilizadas outras alternativas de cura. Ademais, na pesquisa de Saha et al. ${ }^{27}$, os objetivos do uso de medicinas alternativas por estudantes de farmácia, em Bangladesh, estiveram relacionados, em sua maioria, ao tratamento de agravos à saúde menos complexos. Do mesmo modo, Jamshed et al. ${ }^{40}$ encontraram majoritariamente, entre estudantes de farmácia da Malásia, a ideia de que essas terapias podem ser utilizadas para 'doenças menores'.

Incluídas entre as medicinas alternativas ${ }^{\mathbf{1 0}}$, com relação à utilização das práticas religiosas, vale destacar que a maioria dos estudantes afirmou ter religião, sendo a católica a predominante. As práticas compreendidas nos sistemas da medicina popular brasileira podem apresentar contorno religioso, destacando-se as plantas medicinais, utilizadas para fins terapêuticos e religiosos ${ }^{41}$. Segundo Laplantine ${ }^{\mathbf{1}}$, a cultura cristã impregnou os comportamentos médicos ocidentais, bem como a representação da doença, como um mal ou uma punição. Fica evidente, portanto, a influência do sistema cultural-simbólico-religioso nas representações sobre doença e nas práticas de cuidado.

Acerca do uso de pilates, fisioterapia e yoga, é importante lembrar que os estudantes 
consideraram que as doenças podem ser prevenidas. Desse modo, essas práticas, que buscam cuidar do corpo, apresentam aspectos segundo os quais são consideradas como promotoras da saúde. Além disso, a yoga já está sendo incorporada pela PNPIC, episódio que aponta para sua difusão dentro do SUS ${ }^{15}$.

A homeopatia e a acupuntura, que estão dispostas na PNPIC desde a sua publicação, em 2006, com experiências de uso dentro do sistema público de saúde ${ }^{\mathbf{1 2}}$, aparecem na sequência de usos. A homeopatia no Brasil é visualizada e legitimada como uma especialidade médica, possuindo uma intersecção no saber médico ocidental, enquanto que a acupuntura, aproximada da medicina contemporânea desde o século passado, também tem sido prescrita e executada por profissionais de saúde ${ }^{12}$. Em estudos realizados na Espanha e

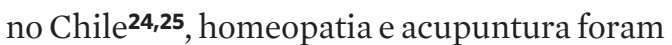
apontadas por estudantes como as terapias alternativas mais conhecidas. Em outra pesquisa com estudantes de medicina no Brasil, as Práticas Não Convencionais em Saúde mais conhecidas eram yoga, homeopatia, acupuntura e práticas religiosas ${ }^{17}$, resultado semelhante ao observado entre os discentes do BIS.

\section{Significados conferidos às Práticas Integrativas e Complementares}

Nesta pesquisa, estudantes atribuem significados positivos às PIC, como pode ser visualizado no dendograma a seguir (figura 2).

Figura 2. Dendograma produzido com auxílio do software Iramuteq e gerado por meio da CHD, referente aos significados atribuídos pelos estudantes do BIS da UFBA às Práticas Integrativas e Complementares. Salvador, Bahia, Brasil, 2017

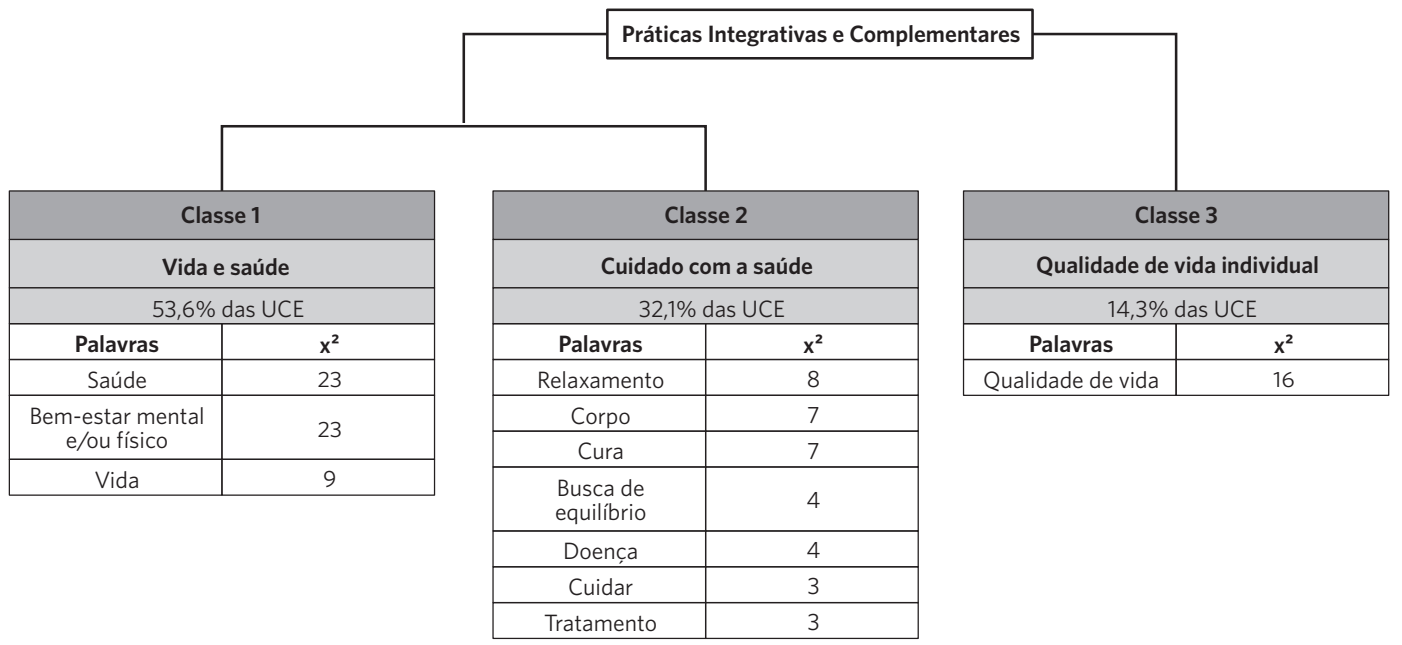

Fonte: Elaboração própria.

A partir do dendograma acima, é possível depreender que a Classe 1 possui palavras que associam as PIC à saúde e à vida; a classe 2 remete aos cuidados com a saúde que podem ser empreendidos por meio das PIC; e a classe 3 apresenta elementos que apontam as PIC como possibilidade de acesso e/ou preservação da qualidade de vida individual.

A classe 1 - Vida e saúde - expõe, por meio dos termos 'saúde', 'bem-estar mental e/ou físico' e 'vida', os sentidos atribuídos às PIC ligados à vida e à saúde. É possível 
compreender, mediante as respostas, que os estudantes expressam benefícios relacionados às racionalidades médicas alternativas enfatizando o bem-estar:

Melhoria da vida e qualidade da saúde. (1-M-19).

Forma de alcançar o bem-estar em vários aspectos da minha vida, não só física. (19-M-30).

Confirmações da minha concepção de vida e saúde. (191-M-23).

[...] são práticas importantes para garantir o bem-estar ao indivíduo que se submete. (219-H-19).

Destarte, infere-se desta última fala que a utilização das PIC está vinculada a uma escolha dos indivíduos. Estes, inseridos num grupo que compreende essas práticas como necessárias para a vida e a saúde, as empregariam, alguns compreendendo que elas possuem outra racionalidade que não a biomédica: "Modelo de busca da saúde alternativa, saindo do modelo biomédico." (190-M-27).

As PIC aparecem, então, como importantes para garantia, manutenção e melhoria da saúde e do bem-estar, nos planos físico e mental, como pode ser observado nos recortes a seguir: “Melhora da saúde física e mental.” (42-H-18); "Importante para o corpo e mente do paciente." (157-H-20).

Ademais, essa classe também exibe as PIC como importantes para o conhecimento do indivíduo sobre si e sobre os processos que atingem a sua vida: "Importantes. A popularização delas ajudará a população a entender mais o processo saúde-doença e como manter a saúde" (119-H-21).

São recursos, portanto, para o empoderamento dos povos, uma vez que promovem o autocuidado de forma integral e potencializam a cidadania em saúde, garantindo a conscientização sobre os procedimentos terapêuticos empreendidos, o que é vital para o SUS, já que sua construção passa pela formação da consciência sanitária ${ }^{12}$.
A classe 2, intitulada Cuidados com a saúde, abrange 'relaxamento', 'corpo', 'cura', 'busca de equilíbrio', 'doença', 'cuidar' e ‘tratamento', determinando pensamentos sobre as PIC como mecanismos para a realização do cuidado com a saúde nos âmbitos físico e mental. No entanto, os estudantes delimitam um sentido particular para o uso das PIC, como se elas atendessem a demandas específicas relacionadas à doença, que diferem das trabalhadas por outras racionalidades médicas:

Formas que algumas pessoas procuram para um determinado processo de cura. (47-M-23).

Formas alternativas visando outras causas que influenciaram nos meus sintomas. (56-H-20).

Essa classe, portanto, apresenta mais concretamente os benefícios que os acadêmicos atribuem a essas práticas (relaxamento, tratamento, cura). Entre elas, o relaxamento aparece como a prática mais recorrente. Nesse sentido, algumas respostas demonstraram a existência de estresse entre os participantes (o que corresponde à presença de agravos psíquicos nas representações sobre doença), sendo as PIC um modo de contornar esse problema:

Forma da pessoa extravasar, desestressar. (127-M-18).

Cuidar não só da matéria do corpo, como também da alma, da mente. (139-M-30).

Formas diferentes de atingir o relaxamento. (146-H-18).

Em estudo realizado com pacientes crôni$\cos ^{42}$, observou-se efeito das PIC no tratamento de sintomas como estresse e dores no corpo.

Nessa classe, também emerge o entendimento das PIC como mecanismos de retomar o equilíbrio do corpo e da mente. $\mathrm{O}$ fato de as representações de doença dos estudantes estarem ancoradas e atreladas à ideia de desequilíbrio insinua a possibilidade de 
intervenção dessas racionalidades médicas sobre as doenças:

Restabelecem o equilíbrio do indivíduo de acordo com a crença de cada um. (33-M-26).

Métodos utilizados para levar o indivíduo a meIhorar algum aspecto que esteja perturbado. (93-H-19).

Ademais, surgiram respostas que apontaram que o uso das PIC é uma forma de contrariar o consumo de medicamentos, movimento muitas vezes ligado à negação da medicalização da vida e da existência: "Forma de não recorrer sempre a medicamentos" (92-M-20); "[...] lembrar que remédio nem sempre resolve tudo" (211-M-18).

Os discentes apontam as PIC também como métodos de prevenção de doenças, não as reduzindo ao contexto físico: "[...] prevenção de doenças físicas e mentais” (34-M-22).

Por conseguinte, nota-se que as classes $1 \mathrm{e}$ 2 compartilham a ideia de que as PIC incidem sobre a vida e a saúde das pessoas que as utilizam, sendo, portanto, parte das vivências culturais das mesmas, as quais, para alguns, antagonizam-se às práticas de saúde instituídas e prevalentes socialmente. Entretanto, essas classes demonstram contraste, na medida em que a primeira confere às PIC ações no sentido geral da vida/saúde, e a segunda as restringe ao tratamento e à prevenção da doença.

Na classe 3, denominada Qualidade de vida individual, está disposta a expressão 'qualidade de vida', demonstrando que esse grupo compreende as PIC como um caminho possível para alcançar a qualidade de vida, porém, numa perspectiva individual, como observado nos excertos a seguir:

Cada indivíduo tem a oportunidade de escolher o que te faz bem [...]. (60-H-20).

Métodos alternativos que sejam capazes de melhorar a qualidade de vida do indivíduo. (189-H-23).
Mesmo que atualmente a qualidade de vida esteja relacionada às condições mais gerais de vida, tocando nas 'causas das causas' dos processos de saúde-adoecimento-cuidado ${ }^{\mathbf{1 4}}$, as ideias dos estudantes não explicitam ligação com os determinantes sociais da saúde. Desse modo, as PIC são compreendidas como medidas terapêuticas individuais, não coletivas. Sabe-se, porém, que elas são oferecidas prioritariamente nos pontos de cuidado dedicados à atenção básica à saúde ${ }^{22}$, nos quais, por meio de medidas gerais e inespecíficas, busca-se promover a saúde e prevenir doenças ${ }^{\mathbf{1 2}}$.

É observado, por conseguinte, que os discentes sugerem nas três classes que as PIC possuem eficácia e são vias para promoção, proteção e recuperação da saúde. As respostas apontam que eles possuem atitudes e imagens positivas sobre essas práticas, além de informações (populares e científicas) que embasam a construção de suas representações. A esse respeito, a literatura corrobora tal achado. Por exemplo, em um estudo ${ }^{18}$ com discentes de medicina, no início do curso, mais da metade declarou que indicaria ou apoiaria a utilização das PIC em pacientes e parentes. Em outro trabalho ${ }^{23}$, também com estudantes de medicina, houve a tomada de posição positiva no tocante à realização de tratamentos de doenças com uso da homeopatia. Em uma pesquisa ${ }^{20} \mathrm{com}$ acadêmicos de enfermagem, estes foram unânimes em afirmar que utilizam as plantas medicinais no dia a dia, contribuindo para a valorização e a disseminação da prática.

É relevante observar que o fato de os estudantes conferirem significados positivos às PIC demonstra que concebem e reconhecem que elas proporcionam benefícios e que acreditam nos seus poderes terapêuticos, além de evidenciar a abertura para que possam ser utilizadas por profissionais de saúde. Ressalta-se, entretanto, que a maioria dos participantes apontou estar no primeiro semestre do BIS e encontrar-se na faixa etária entre 18 e 24 anos, ou seja, no início 
do percurso formativo, ao longo do qual terão a possibilidade de engrandecer ainda mais esses pensamentos e práticas. Essa perspectiva corresponde aos resultados da pesquisa desenvolvida por Feitosa et al. ${ }^{19}$, os quais evidenciam que o interesse pelas plantas medicinais foi maior em estudantes concluintes que em recém-ingressos. No trabalho de Contreras et al. ${ }^{25}$, no entanto, as opiniões desfavoráveis com relação às terapias complementares aumentaram com a progressão acadêmica.

Outro ponto que merece destaque está relacionado à oferta, ao corpo discente, da possibilidade de apreender e tecer reflexões acerca das PIC, pois pesquisas demonstram o despertar do interesse entre estudantes da área da saúde em estudar sobre elas 16,19,22. O BIS, por exemplo, oferece tanto componentes curriculares ${ }^{\mathbf{4 3}}$ quanto atividades de extensão ${ }^{\mathbf{4 4}}$, que abordam as PIC de maneira geral ou específica. Logo, o escopo de assimilação das PIC nesse curso propicia tanto o contato teórico quanto prático. $\mathrm{O}$ reconhecimento e a aceitação das PIC por profissionais da saúde têm sido apontados como determinantes para o acesso dos usuários ${ }^{\mathbf{4 2}}$.

O curso oferece, portanto, informações sobre racionalidades médicas alternativas que podem subsidiar o reforço de atitudes positivas com relação a elas e a promoção da sua difusão entre os estudantes. É provável, então, que isso, por incidir nas três dimensões estruturantes das representações sociais - informação, campo da representação e atitude -, seja capaz de fortalecer as representações sobre doença, para além do modelo biomédico.

\section{Considerações finais}

As representações sociais de doença relacionadas à ideia de desequilíbrio entre os participantes mostram-se associadas ao que pode ser prevenido. Para os discentes, a doença, ao acometer o ser humano, deve ser tratada, já que, ao se constituir como algo ruim, precisa ter um fim. Essas representações se encontram em estreita consonância com o que é definido pela medicina contemporânea ocidental. Além disso, os estudantes acreditam no poder das PIC, mencionando os seus benefícios e, em parte, o seu uso. Os significados atribuídos por elas/eles às PIC caminham no sentido da preservação e do cuidado com a saúde. Esse aspecto é relevante, considerando-se a necessidade de formar trabalhadores da saúde conscientes e comprometidos no que diz respeito a essas racionalidades, demandadas pela população brasileira nos serviços de saúde. Não obstante, ainda é o pensamento biologicista que domina as práticas de cuidado, promoção, proteção e recuperação da saúde.

Os resultados deste estudo apontam para a existência de representações de doença predominantemente atreladas à racionalidade da biomedicina, dominante no ocidente, ao mesmo tempo que revelam uma abertura para outros sistemas médicos. Como a maior parte dos participantes cursava o primeiro semestre do curso no momento da coleta de dados, é possível que o uso de PIC e os significados atribuídos a elas sejam enriquecidos ao longo da trajetória acadêmica.

Apreender as representações sociais sobre a doença e os usos e significados conferidos às PIC é um recurso para o entendimento dos projetos terapêuticos empreendidos nos serviços de saúde, balizados ou não por pensamentos hegemônicos. Isso possibilita a compreensão e o fortalecimento de políticas públicas ligadas à saúde, bem como o entendimento de práticas de cuidado difundidas entre os brasileiros, relacionadas à cultura e emergentes do meio social. Nesse sentido, realizar um estudo sobre esses aspectos caminha na direção da execução de um cuidado integral à saúde das pessoas, já que expõe a existência de mais de uma racionalidade médica, determinando, assim, que as ações ligadas à saúde podem ser realizadas em consonância com as concepções aceitas nos grupos sociais, sendo mais eficazes quando assim são feitas. 


\section{Colaboradores}

Coelho MTAD (0000-0001-7857-7473)* contribuiu para a concepção, o planejamento, a análise e a interpretação dos dados; revisão crítica do conteúdo; e aprovação da versão final do manuscrito. Carvalho VP
(0000-0001-5249-2818)* contribuiu para a concepção, o planejamento, a análise e a interpretação dos dados; revisão crítica do conteúdo. Porcino C (0000-0001-6392-0291)* contribuiu para a concepção, o planejamento, a análise e a interpretação dos dados; revisão crítica do conteúdo.

\section{Referências}

1. Laplantine F. Antropologia da doença. 4. ed. São Paulo: WMF Martins Fontes; 2010.

2. Minayo MCS. Palavras, interações e representações sociais. In: Minayo MCS. O desafio do conhecimento: pesquisa qualitativa em saúde. 12. ed. São Paulo: Hucitec; 2010. p. 204-60.

3. Luz MT. Comparação de representações de corpo, saúde, doença e tratamento em pacientes e terapeutas de homeopatia, acupuntura e biomedicina na rede de saúde do município do Rio de Janeiro. In: Luz MT, Barros NS, organizadores. Racionalidades médicas e práticas integrativas em saúde: estudos teóricos e empíricos. Rio de Janeiro: UERJ; 2012. p. 217-47.

4. Canguilhem G. O normal e o patológico. Rio de Janeiro: Forense Universitária; 1995.

5. Durkheim E. As regras do método sociológico. 3. ed

São Paulo: Martins Fontes; 2007.

*Orcid (Open Researcher and Contributor ID).
6. Moscovici S. A psicanálise, sua imagem e seu públi- co. Petrópolis: Vozes; 2012.

7. Moscovici S. Das representações coletivas às representações sociais: elementos para uma história. In: Jodelet D, organizadora. As representações sociais. Rio de Janeiro: EdUERJ; 2001. p. 45-66.

8. Herzlich C. A problemática da representação social e sua utilidade no campo da doença. Physis. 1991; $1(2): 23-36$.

9. Cardoso MHCA, Gomes R. Representações sociais e história: referenciais teórico-metodológicos para o campo da saúde coletiva. Cad. Saúde Pública. 2000; 16(2):499-506.

10. Luz MT. Cultura Contemporânea e Medicinas Alternativas: novos paradigmas em saúde no fim do século XX. Physis. 1997; 7(1):13-43.

11. Camargo Jr. KR. The biomedicine. Physis. 2005; 15(suppl):177-201. 
12. Brasil. Ministério da Saúde. Política Nacional de Práticas Integrativas e Complementares no SUS. Brasília, DF: Ministério da Saúde; 2006.

13. Barros NF, Spadacio C, Costa MV. Trabalho interprofissional e as Práticas Integrativas e Complementares no contexto da Atenção Primária à Saúde: potenciais e desafios. Saúde debate. 2018; 42(espl):163-173.

14. Paim JS. O que é o SUS. Rio de Janeiro: Fiocruz; 2009.

15. Brasil. Ministério da Saúde. Portaria ${ }^{\circ} 849$, de 27 de março de 2017. Inclui a Arteterapia, Ayurveda, Biodança, Dança Circular, Meditação, Musicoterapia, Naturopatia, Osteopatia, Quiropraxia, Reflexoterapia, Reiki, Shantala, Terapia Comunitária Integrativa e Yoga à Política Nacional de Práticas Integrativas e Complementares. Diário Oficial da União. 28 Mar 2017.

16. Brasil. Ministério da Saúde. Portaria $n^{\circ} 702$, de 21 de março de 2018. Altera a Portaria de Consolidação ${ }^{0}$ 2/GM/MS, de 28 de setembro de 2017, para incluir novas práticas na Política Nacional de Práticas Integrativas e Complementares - PNPIC. Diário Oficial da União. 22 Mar 2018.

17. Külkamp IC, Burin GD, Souza MHM, et al. Aceitação de práticas não-convencionais em saúde por estudantes de medicina da Universidade do Sul de Santa Catarina. Rev. bras. educ. méd. 2007; 31(3):229-235.

18. Morales NM, Min LS, Teixeira JEM. Atitude de Estudantes de Medicina frente a Terapias Alternativas e Complementares. Rev. bras. educ. méd. 2015; 39(2):240-245.

19. Feitosa MHA, Soares LL, Borges GA, et al. Inserção do Conteúdo Fitoterapia em Cursos da Área de Saúde. Rev. bras. educ. méd. 2016; 40(2):197-203.

20. Badke MR, Heisler EV, Ceolin S, et al. O conhecimento de discentes de enfermagem sobre uso de plantas medicinais como terapia complementar. Rev. pesqui. cuid. fundam. 2017; 9(2):459-465.

21. Carnevale RC, Brandão AL, Ferraz RO, et al. O Ensino da Acupuntura na Escola Médica: Interesse e Desconhecimento. Rev. bras. educ. méd. 2017; 41(1):134-44.

22. Tesser CD, Sousa IMC, Nascimento MC. Práticas Integrativas e Complementares na Atenção Primária à Saúde brasileira. Saúde debate. 2018; 4(1):174-188.

23. Teixeira MZ. Homeopatia: desinformação e preconceito no ensino médico. Rev. bras. educ. méd. 2007; 31(1):15-20.

24. Ballesteros-Peña S, Fernández-Aedo I. Conocimientos y actitudes sobre terapias alternativas y complementarias en estudiantes de ciencias de la salud. Investigación educ. médica. 2015; 4(16):207-215.

25. Contreras D, Alamos MJ, Chang M, et al. Opiniones sobre terapias complementarias por parte de los estudiantes de medicina de la Pontificia Universidad Católica de Chile. Año 2014. Rev. méd. Chile. 2015; 143(8):1020-1027.

26. Flaherty G, Fitzgibbon J, Cantillon P. Attitudes of medical students toward the practice and teaching of integrative medicine. J. Integr. Med. 2015; 13(6):412415 .

27. Saha, BL, Seam MOR, Islam MM, et al. General Perception and Self-Practice of Complementary and Alternative Medicine (CAM) among Undergraduate Pharmacy Students of Bangladesh. BMC complement. altern. med. 2017; 17(314):1-8.

28. Gomes R, Mendonça EA. A representação e a experiência da doença: princípios para a pesquisa qualitativa em saúde. In: Minayo MCS, Deslandes SF, organizadoras. Caminhos do pensamento: epistemologia e método. Rio de Janeiro: Fiocruz; 2002. p. 109-32.

29. Rapaport D, Schafer R, Gill M. Testes de diagnóstico psicológico. Buenos Aires: Editorial Paidós; 1971.

30. Di Giacomo JP. Aspects méthodologiques de l'analyse des répresentations sociales. Cahiers de Psychologie Cognitive 1981; 1(1):397-422.

31. Nóbrega SM, Coutinho MPL. A técnica de associa- 
ção livre de palavras. In: Coutinho MPL, Saraiva ERA. Métodos de pesquisa em psicologia social: perspectivas qualitativas e quantitativas. João Pessoa: Editora Universitária; 2011. p. 95-106.

32. Neves DAB, Brito RC, Códula ACC, et al. Protocolo Verbal e Teste de Associação Livre de Palavras: perspectivas de instrumentos de pesquisa introspectiva e projetiva na ciência da informação. Ponto Acesso. 2014; 8(3):64-79.

33. Ratinaud P, Marchand P. Application de La méthode ALCESTE à de "gros" corpus et stabilitédes "mondes lexicaux": Analyse du "Cable Gate" avec IraMuTeQ. In: Actes des 1leme Journées Internationales d'Analyse Statistique des Données Textuelles; 2012; Liège: JADT; 2012. p. 835-44.

34. Pestana MH, Gageiro JN. Análise de dados para ciências sociais: a complementaridade do SPSS. 6. ed. Lisboa: Edições Sílabo; 2014.

35. Oliveira DC, Gomes AMT, Marques SC. Análise estatística de dados textuais na pesquisa das representações sociais: alguns princípios e uma aplicação ao campo da saúde. In: Menin MSS, Schimizu AM, organizadores. Experiência e representação social: questões teóricas e metodológicas. São Paulo: Casa do psicólogo; 2005. p. 157-200.

36. Brasil. Ministério da saúde. Resolução $n^{\circ} 466$, de 12 de dezembro de 2012. Diário Oficial da União. 12 Dez 2012.

37. Universidade Federal da Bahia. Projeto Pedagógico do Bacharelado Interdisciplinar em Saúde. Salvador: IHAC/UFBA; 2010.

38. Coelho MTÁD, Santos VP, Silva BM, et al. Concepções e práticas de saúde e doença entre alunos da primeira turma do Bacharelado Interdisciplinar em
Saúde da Universidade Federal da Bahia. In: Teixeira CF, Coelho MTÁD, organizadoras. Uma experiência inovadora no ensino superior: bacharelado interdisciplinar em saúde. Salvador: EDUFBA; 2014. p. 111126.

39. Conselho Federal de Psicologia. Subsídios para a campanha Não à medicalização da vida: medicalização da educação. Brasília, DF: CFP; 2012.

40. Jamshed SQ, Khan MU, Ahmad A, et al. Knowledge, Perceptions, and Attitudes toward Complementary and Alternative Medicines among Pharmacy Students of a Malaysian Public University. J. Pharm. \& Bioallied. Sci. 2016; 8(1):34-38.

41. Camargo MTLA. As plantas medicinais e o sagrado, considerando seu papel na eficácia das terapias mágico-religiosas. Revista Nures. 2014; Ano X(26):1-16.

42. Dacal MPO, Silva IS. Impactos das práticas integrativas e complementares na saúde de pacientes crônicos. Saúde debate. 2018; 42(118):724-735.

43. Teixeira CFS, Coelho MTAD, Rocha MND. Bacharelado interdisciplinar: uma proposta inovadora na educação superior em saúde no Brasil. Ciênc. Saúde Colet. 2013; 18(6):1635-1646.

44. Veras RM, Cardoso GMP, Figueredo WN, et al. Bacharelado Interdisciplinar em Saúde: um mapeamento de suas ações extensionistas no período de 2009 a 2012. In: Coelho MTÁD, Teixeira CF, organizadoras. Problematizando o Campo da Saúde: Concepções e práticas no bacharelado interdisciplinar. Salvador: EDUFBA; 2017. p. 231-48.

Recebido em 26/12/2018

Aprovado em 28/04/2019

Conflito de interesses: inexistente

Suporte financeiro: Conselho Nacional de Desenvolvimento

Científico e Tecnológico (CNPq) 